\section{Chronic infection double-whammy}

The role of regulatory $T$ cells ( $T_{\text {reg }}$ cells) in sustaining chronic viral infection is unclear. In the Journal of Experimental Medicine, Ahmed and colleagues address this question by combining an inducible $T_{\text {reg }}$ cell-deletion system with the chronically infectious lymphocytic choriomeningitis virus clone 13 . During chronic infection, cytotoxic T cells adopt a poorly functional 'exhausted' phenotype, but depletion of $T_{\text {reg }}$ cells substantially restores both functionality and proliferative potential in a costimulationdependent manner. However, this recovery does not result in clearance of the virus, since these cytotoxic T cells still express the inhibitory molecule PD-1 and infected cells upregulate its ligand, PD-L1. A combination of PD-L1 and depletion of $T_{\text {reg }}$ cells effectively clears the virus and is superior to blockade of PD-L1 alone. Carefully defined depletion of $\mathrm{T}_{\text {reg }}$ cells in conjunction with blockade of PD-L1 may therefore optimize the immunotherapy of chronic infection while minimizing potentially harmful side effects such as the unmasking of autoimmunity.

J. Exp. Med. (11 August 2014) doi:10.1084/jem.20132577

\section{ChIP to the next level}

The classic molecular model of cellular differentiation suggests that there is progressive closing of chromatin as cells transition from a progenitor state to a terminally differentiated state. In Science, Amit and colleagues develop a novel approach to chromatin immunoprecipitation (ChIP) called 'iChIP' that enables ChIP to be applied to only a few hundred cells. Notably, this level of sensitivity allows the examination of rare intermediate cell populations that are otherwise inaccessible to standard approaches. iChIP is used to examine the chromatin dynamics of enhancers and promoters in developing hematopoietic stem cells. A poised enhancer repertoire is established early in development and before the expression of transcriptional programs in differentiated cells. Collectively, the data present a modified picture of hematopoiesis, with enhancer potential peaking not at the stem cell stage but in the more oligoclonal progenitor cells.

Science (7 August 2014) doi:10.1126/science.1256271

\section{Restricting plasma cells}

The number of long-lived antibody-producing plasma cells is typically kept low, which has been interpreted as evidence suggesting extrinsic constraints on the niches that harbor such cells. In Science Signaling, Infantino et al. reveal that the tyrosine kinase Lyn exerts intrinsic control of plasma cell numbers by regulating their survival. Mice that lack $B$ cell expression of Lyn accumulate a greater number of splenic IgM $^{+}$plasma cells by prolonging their survival. Lyn acts to suppress cytokine signaling that occurs by IL-6-STAT3 and IL-3-STAT5 pathways, both of which induce expression of the survival factor Mcl-1. Lyn does not alter signaling via other survival factors, including APRIL, BAFF and IL-21. These findings suggest that Lyn deficiency could contribute to some autoimmune diseases, such as systemic lupus erythematosus, that are characterized by excessive numbers of plasma cells.

Sci. Signal. (12 Aug 2014) doi:10.1126/scisignal.2005105
$L A D$

\section{Antigen-presenting ILCS}

Innate lymphoid cells that produce type 2 cytokines (ILC2 cells) precede and are known to influence expansion of the adaptive $\mathrm{CD} 4^{+}$type 2 helper $\mathrm{T}$ cell $\left(\mathrm{T}_{\mathrm{H}} 2\right.$ cell) responses. In Immunity, McKenzie and colleagues use two different ILC2 cell-deficient mouse strains to show that ILC2 cells contribute to the development of efficient $\mathrm{T}_{\mathrm{H}} 2$ responses to the parasitic worm Nippostrongylus brasiliensis in a manner dependent on major histocompatibility complex (MHC) class II. ILC2 cells express MHC class II and the costimulatory receptors CD80 and CD86, acquire and process antigen and can induce antigen-specific activation and proliferation of $\mathrm{T}$ cells with efficiency lower than that of $\mathrm{CD} 11 \mathrm{c}^{+}$ dendritic cells but equivalent to that of plasmacytoid dendritic cells or naive $B$ cells. The antigen-dependent crosstalk with $\mathrm{T}$ cells leads to the proliferation of ILC2 cells and secretion of type 2 cytokines in a manner dependent on the production of interleukin 2 (IL-2) from T cells. Human ILC2 cells express MHC class II and can also present antigen to T cells, which suggests a conserved mechanism.

Immunity 41, 283-295 (2014)

\section{Receptor for new PAMPs}

The aryl hydrocarbon receptor ( $\mathrm{AhR}$ ) is a ligand-dependent transcription factor that senses environmental toxins and endogenous ligands to induce detoxifying enzymes and modulate differentiation of cells of the immune system. In Nature, Kaufmann and colleagues show that AhR is a receptor for bacterial pigments and induces the production of cytokines and chemokines as part of the antibacterial immune response. Pigmented virulence factors such as phenazines from Pseudomonas aeruginosa bind AhR and activate the detoxification pathway. During infection of mice with $P$. aeruginosa, AhR signaling is required in neutrophils and macrophages as well as in respiratory epithelial cells for the induction of proinflammatory cytokines and chemokines, neutrophil recruitment and mucus production. AhR-deficient mice are more sensitive to infection with $P$. aeruginosa and Mycobacterium tuberculosis. Thus, AhR functions as an intracellular pattern-recognition receptor for bacterial pigments, which can be defined as a new class of pathogen-associated molecular patterns.

IV

Nature (13 August 2014) doi:10.1038/nature13684

\section{Interferons in the brain}

Immunological contributions are increasingly associated with cognitive deficiencies that can occur during aging. In Science, Baruch et al. report that older mice exhibit altered expression profiles of genes encoding interferons in the choroid plexus, which acts as an interface between blood and the central nervous system, relative to that of younger mice. Aged mice have lower expression of genes responsive to interferon- $\gamma$ but higher expression of genes dependent on type I interferons. This type I interferon signature is not altered in young-old parabiont mouse couplings. Similar expression profiles of genes encoding type I interferons are seen in postmortem sections of human brains. Notably, these differences in interferon response are accompanied by changes in choroid plexus function to regulate leukocyte trafficking and influence brain plasticity, as revealed in cognitive memory tests. What underlies the higher expression of type I interferons in the choroid plexus remains to be determined.

$L A D$

Science (21 August 2014) doi:10.1126/science.1252945 